\title{
EXPRESSION OF BONE MORPHOGENETIC PROTEIN- 2 UNDER SIMVASTATIN THERAPY AFTER CYCLOSPORIN -A- INDUCED ALVEOLAR BONE LOSS IN RATS
}

\begin{abstract}
Samir $\mathrm{H}^{1} B D S$, Shafik $\mathrm{S}^{2} P h D$, Yahia $\mathrm{M}^{3} P h D$, Mamdouh $\mathrm{N}^{4} P h D$.
Abstract:

Introduction: Cyclosporin-A- has been used as an immunosuppressant to prevent the rejection of organ transplants. However, alveolar bone loss is an important negative side-effect of this drug. Simvastatin, a hydroxy-3-methylglutaryl coenzyme A (HMG-CoA) reductase inhibitor, is known to inhibit cholesterol biosynthesis. It has advanced effects on bone formation in vivo and in vitro. So, we evaluated the expression of BMP 2 after administration of simvastatin in cyclosporin -A-associated alveolar bone loss in rats.

Objective: To evaluate the effect of simvastatin and cyclosporin -A- on Alveolar bone by investigating the expression of Bone Morphogenetic Protein -2 (BMP-2) using Immunohistochemical and Image analysis investigation methods.

Materials and methods: 24 adult male albino rats were divided into 3 groups: Group I: control group; 4 rats, Group II: cyclosporine -A- group; 10 rats $(10 \mathrm{mg} / \mathrm{kg})$ daily subcutaneous injection, Group III: Cyclosporin -A /Simvastatin group; 10 rats, simvastatin was taken orally daily $(20 \mathrm{mg} / \mathrm{kg} /$ day). Two rats from the control group and 5 rats from each of the studied experimental groups (group II \& III) were sacrificed on days 15 and 30 consecutively, and examined using Immunohistochemical method, and Image analysis.

Results: Immunohistochemical results revealed strong expression of BMP 2 in osteoblasts, osteocytes after simvastatin administration and weak expression in CsA. The same results were statistically significant in Immunohistochemical Optical Density (IOD) results. Histomorphometrical analysis of bone volume showed a significant increase in bone volume in simvastatin group than CsA group, and significant decrease in CsA than control.

Conclusion: we can conclude that Simvastatin counteract the adverse effect of CsA induced alveolar bone loss by induction of BMP 2 in osteoblasts and osteocytes that induced new bone formation.

Keywords: Simvastatin, Cyclosporin A, bone loss, Bone Morphogenitic Protein 2.

1- Master student at the Oral Biology Department, Faculty of Dentistry, Alexandria University

2- Professor of Oral Biology, Faculty of Dentistry, Alexandria University

3- Professor of Histochemistry and Cell Biology, Medical Research Institute, Alexandria University

4- Lecturer of Oral Biology, Faculty of Dentistry, Alexandria University
\end{abstract}

\section{INTRODUCTION}

Bone is a rigid and dynamic organ that is characterized as a type of specialized connective tissue. Biochemically, it is defined by a mixture of inorganic elements and an organic matrix. It is composed of 50 to $70 \%$ mineral, 20 to $40 \%$ organic matrix, 5 to $10 \%$ water, and $3 \%$ lipids (1).

Bone matrix is mostly composed of type I collagen, about $90 \%$, with trace amounts of types III and V. and $10 \%$ ground substances including: proteoglycan, glycoproteins, and non-collagenous proteins, that are embedded in the extracellular matrix as (bone morphogenetic proteins, insulin like growth factor-1 and 6, colony stimulating factors, cytokines, adhesion molecules) (2).

Bone remodeling is the process by which bone is renewed to maintain bone strength and mineral homeostasis. The remodeling process resorbs old bone and forms new bone to prevent accumulation of bone micro damage. Remodeling begins before birth and continues until death (3).

The remodeling cycle is composed of four sequential phases which are: quiescent, activation, resorption, reversal, and formation phases (4).
Bone Morphogenetic Protein (BMPs) are a group of proteinaceous growth factors in the TGF- $\beta$ superfamily (5). The discovery of BMPs in the pioneering work by Urist in 1965 (6) was a landmark in the development of bone tissue engineering. The classical role for BMPs is considered to be the induction of (ectopic) cartilage and bone formation (6).

According to the WHO, Osteoporosis is defined as "a systemic skeletal disease characterized by low bone mass and microarchitectural deterioration of bone tissue with a consequent increase in bone fragility and susceptibility to fracture". It is a common and costly disease with a considerable annual health system burden all over the world. Morbidity and mortality related to this disease and its associated fragility fractures are also increasing (7).

In osteoporosis, disruption of the resorption and formation processes in the bone remodeling cycle results in net bone loss and, therefore, in a lower bone mass and increased risk of fracture (8).

Osteoporosis affects the jawbones, whose structure may be impaired by other conditions resulting in bone loss. One of these is periodontitis (PD), a chronic infection-mediated condition modulated by different genetic and environmental 
factors, characterized, in advanced forms, by loss of the soft tissue attachment to teeth and resorption of alveolar bone (9).

Osteoporosis is a significant adverse reaction in transplant recipients. Recently, an increasing number of immunosuppressive programs use glucocorticoid-free regimens, but other immunosuppressants, such as calcineurin inhibitors (e.g. cyclosporine A), are also associated with the pathogenesis of transplantation-related osteoporosis (10).

Cyclosporin-A- (CsA) is a cyclical polypeptide of 11 amino acids, one of which is unique to the cyclosporins. First isolated as an antifungal agent, it has been shown to have marked immunomodulatory properties. These properties have meant that the drug can be used as an immunosuppressant to prevent the rejection of transplants, both of organs (kidney, liver, and pancreas) and bone marrows, as well as in the control of autoimmune diseases (11). One of the proposed side effects of cyclosporine is bone loss after long-term use. Bone loss was seen in trabecular bone more than cortical bone (12).

CyclosporineA inhibits osteoblast differentiation, osteocalcin production, and collagen synthesis, reducing the bone replaced in each remodeling cycle (13).

Simvastatin is a chemically modified derivative of lovastatin. It is a butanoic acid with the empirical formula C25H38O5 (14).

Simvastatin is a reversible competitive inhibitor of the enzyme 3-hydroxy-3-methylglutaryl coenzyme A reductase (HMG-CoA reductase), so, like the other statins, is an inhibitor of the mevalonate pathway and consequently cholesterol synthesis (15).

Simvastatin, a synthetic statin has a number of pleiotropic effects as well. In addition to its anti-resorptive actions, it has been found to exert anabolic effects on bone (16).

Local stimulation of BMP-2, a major bone growth regulatory factor, can lead to new bone formation. Mundy et al. (1999) (17) identified that lovastatin, and simvastatin, mevastatin, and fluvastatin increased gene expression for BMP-2 in osteoblasts.

The biologically significant anti-inflammatory and antioxidant properties of simvastatin are other pleiotropic effects of interest from a periodontal therapeutic standpoint (18).

One of the most important pleiotropic effect of statins are anticancer agents because of their ability to trigger apoptosis in a variety of tumor cells in a manner that is sensitive and specific to the inhibition of HMG-CoA reductase (19).

\section{MATERIALS AND METHODS Materials}

\section{Experimental animals:}

The study was performed after gaining the approval of the Research Ethics Committee, Faculty of Dentistry, Alexandria University.

Twenty-four adult male albino rats weighting 90-100 grams were selected to be used in this study. These animals were obtained from the Institute of Medical Research, Alexandria University.
Animals were housed in specially designed wire mesh bottom cages. They were supplied a regular diet throughout the whole experimental period which last for 30 days.

Rats were divided randomly into three groups: Group I: control (consisted of 4 rats) was injected with $1 \mathrm{ml}$ saline.

Group II: Cyclosporin -A (Sandimmun®; supplied in form of vials. Each vial contained $50 \mathrm{mg}$ of Cyclosporine A).

The group consisted of 10 rats,(20) which were subcutaneously injected with Cyclosporin -A-, subcutaneous daily injection (10 mg/kg body weight, once a day).

Group III: cyclosporin A/Simvastatin group consisted of 10 rats. This group was treated with both Cyclosporin -A- and Simvastatin (Zocor®; supplied in form of tablets. Each tablet contained $20 \mathrm{mg}$ of simvastatin). Simvastatin was taken by oral daily doses (once a day) at $20 \mathrm{mg} / \mathrm{kg}$ (21).

Two rats from the control group and 5 rats from each of the studied experimental groups (group II \& III) were sacrificed on days 15 and 30 consecutively after commencement of the daily treatments.

\section{Methods:}

\section{Immunohistochemical technique.}

Immunohistochemical procedures were carried out using an indirect immunoperoxidase system. Tissue slices were blocked on the coated slides and treated with rabbit polyclonal antibodies to BMP-2 for detecting the immunostaining by Avidin Biotin Complex (ABC) method (22).

\section{Image analysis}

Morphometric analysis was carried out in alveolar bone of Haematoxylin and Eosin-stained section. Images were viewed and recorded using the Olympus microscope equipped with digital camera, using computer program Matlab software (Image J, the MATHWORKS, Inc., and USA). The image of each section of all groups was captured using $x 40$ objective lens $($ Barr $=50 \mu \mathrm{m})$ with numerical aperture of a high resolution (16 bit digital camera, $1280 \times 1024$ pixel) for counting osteoclast and osteoblast cells and calculating bone volume, and immuohistochemical Optical Density (IOD).

\section{Statistical analysis (23):}

Data were fed to the computer and analyzed using IBM SPSS software package version 20.0. Quantitative data were described using range (minimum and maximum), mean, standard and median. The distributions of quantitative variables were tested for normality using KolmogorovSmirnov test, Shapiro-Wilk test and D'Agstino test, also Histogram and QQ plot were used for vision test. For normally distributed data, comparison between more than two population were analyzed F-test (ANOVA) to be used and Post Hoc test (LSD), For abnormally distributed data, comparison between more than two independent population were done using Kruskal Wallis test, Significance of the obtained results was judged at the $5 \%$ level. 


\section{RESULTS}

The results obtained from this study included both immunohistochemical results and image analysis.

\section{Immunohistochemical findings}

Immunohistochemistry stains BMP 2 protein in the apical region of the alveolar bone of the albino rats was done on the control and the two experimental study groups.

In the present study the BMP 2 immunoreactivity appeared as granules and diffuses brown color in cytoplasm and cell membrane of osteoblasts, osteocytes and bone matrix.

The control sample of the BMP 2 immunoreactivity was negative due to the absence of the polyclonal antibody of BMP 2 immunoreactivity.

\section{Group A (15 days):}

Group IA (Control): (fig 1)

The immunointensity of the cells were moderate $(+2)$ to strong $(+3)$. The bone matrix appeared with several spreading granules (appeared in incremental lines) representing the presence of BMP 2 in the matrix itself.

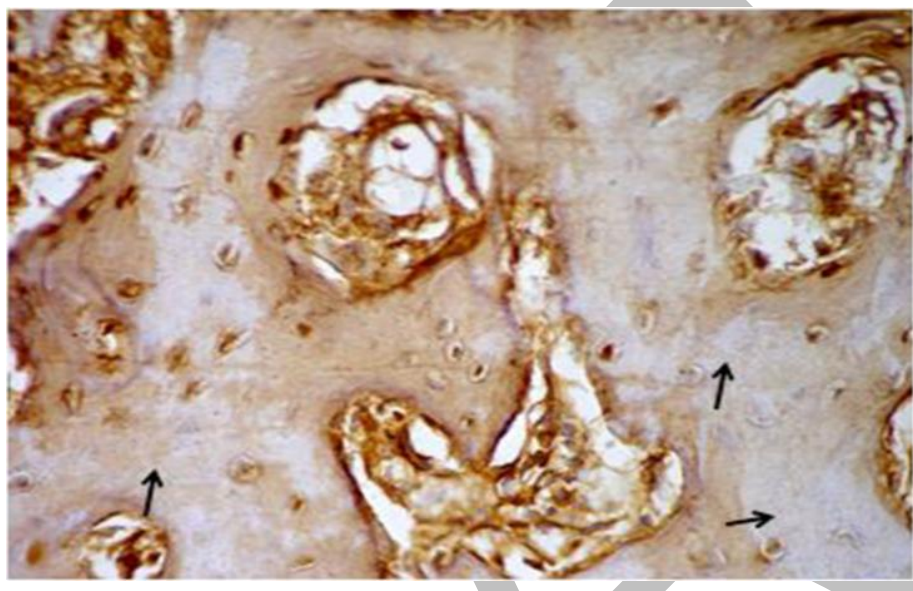

Fig. 1: Paraffin section micrograph of IA (control 15 days) of rat alveolar bone in the apical region. (ABC -DAB Bar $=50 \mu \mathrm{m}$ X400)

Group IIA( CsA 15 days): (fig 2)

The immunoreactive stains of BMP 2 protein were weak $(+1)$ to moderate $(+2)$ on osteoblasts, and osteocytes. The bone matrix appeared with less expression and diffuse granules (This group revealed a decrease immunoreactive expression of BMP 2 protein than group IA) lesser than group IA).

Group IIIA (CsA\& Simvastatin 15 days): (fig 3)

The osteoblast and osteocytes were moderate $(+2)$ to strong $(+3)$ positive expression of immunoreactive stains of BMP 2 protein. The bone matrix appeared with several spreading granules (appeared in incremental lines) representing the presence of BMP 2 protein expression in the matrix itself.

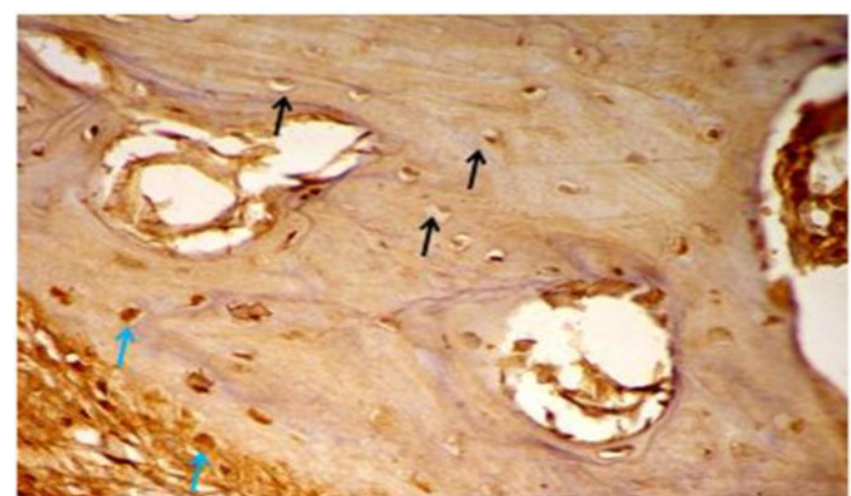

Fig. 2: Paraffin section micrograph of micrograph of rat alveolar bone administrated (CsA 15 days) in the apical region.( $\mathrm{ABC}-\mathrm{DAB} \quad \mathrm{Bar}=50 \mu \mathrm{m}$ X400)

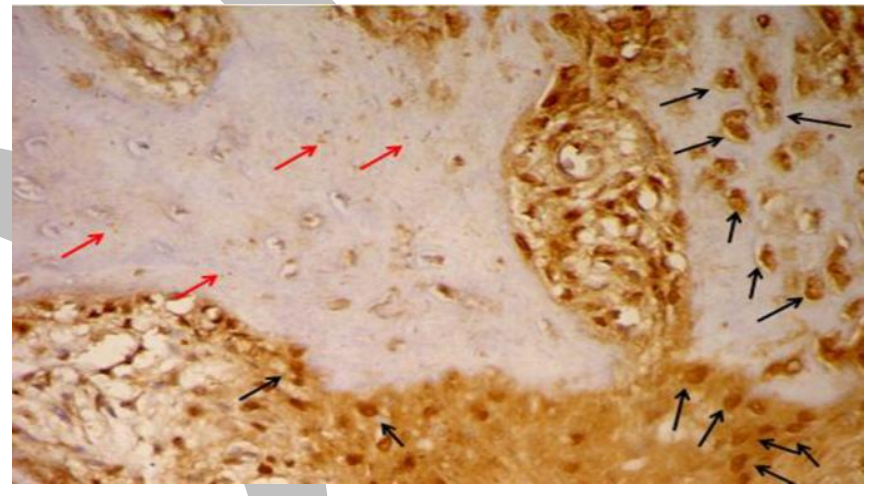

Fig. 3: Paraffin section micrograph of rat alveolar bone administrated (simvastatin \& CsA 15 days) of rat alveolar bone in the apical portion. $(\mathrm{ABC}-\mathrm{DAB}$ Bar $=50 \mu \mathrm{m}(\mathrm{x} 400)$

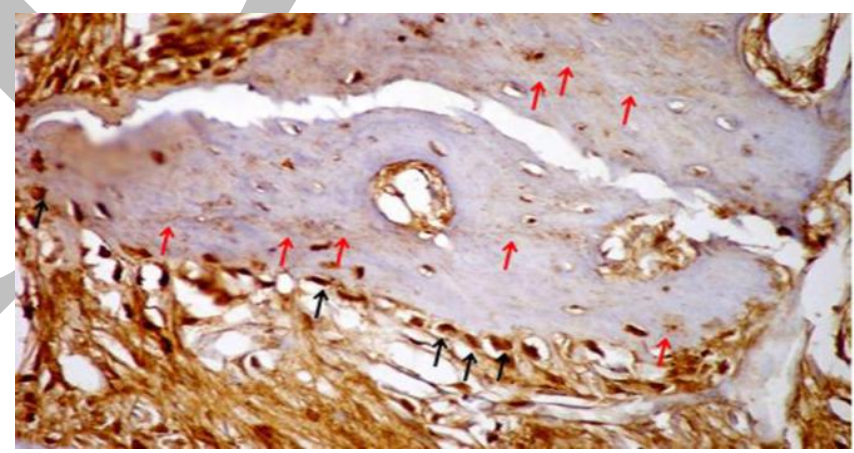

Fig. 4: Paraffin section micrograph of rat alveolar bone of control (30 days) in the apical portion ( $\mathrm{ABC}-\mathrm{DAB}$ Bar $=50 \mu \mathrm{m}(\mathrm{x} 400)$

\section{Group B (30 days):}

Group IB (control): (fig 4)

The immunoreactive of BMP 2 protein expression of the osteoblast cells, and osteocyte were moderate $(+2)$ to strong $(+3)$. The bone matrix appeared with several spreading granules (appeared in incremental lines) representing the presence of BMP 2 protein expressed in the matrix itself.

Group IIB (CsA 30 days): (fig 5)

There was a decreased of the immunoreactive stain of BMP 2 protein expression in the osteoblast cells, osteocytes were given a negative $(0)$ to weak $(+1)$. 


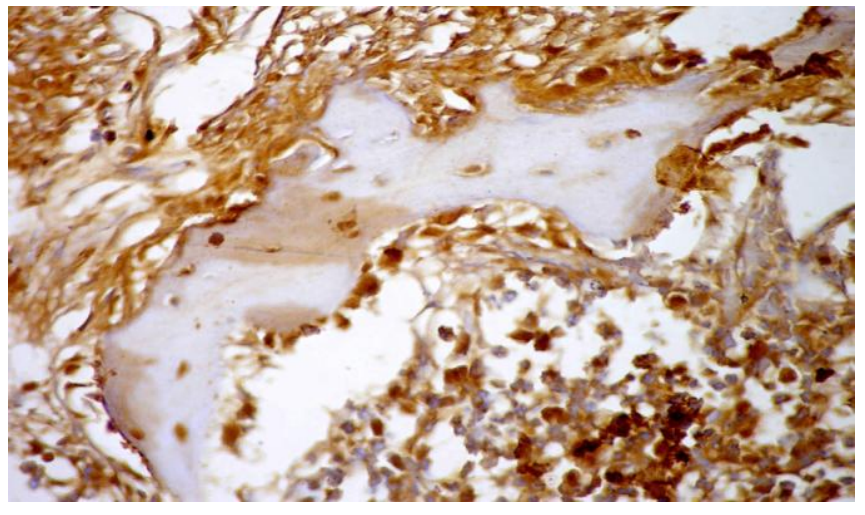

Fig. 5: Paraffin section micrograph of rat alveolar bone administrated (CsA 30 days) in the apical portion. (ABC $-\mathrm{DAB}$ Bar $=50 \mu \mathrm{m} \times 400)$.

Group IIIB (CsA\& Simvastatin 30 days): (fig 6)

The long duration of the administrative simvastatin plus CsA showed an increase of the BMP 2 protein expression. The osteoblast and osteocytes were moderate $(+2)$ to strong $(+3)$ immunoreactive stain. The bone matrix appeared with several spreading granules, representing the presence of BMP 2 protein in the matrix itself. The osteoblasts appeared plump and strongly immunopositive arranged on the bone surface.

\section{Image Analysis:}

a. Bone volume:

\section{Group A (after 15 days)}

Table (1) showed mean values of bone volume per total volume in the apical region of the control (IA), CsA (IIA), and (CsA \&Simvastatin) (IIIA), after 15 days, the values were $(0.21 \pm 0.02,0.12 \pm 0.02,0.26 \pm 0.05)$, respectively.

There was a statistically significant decrease in the bone volume in group CsA group (IIA) in comparison with control group (IA) $(\mathrm{p} 1=0.002)$. But, a statistically significant increase in the bone volume in group (CsA \&Simvastatin) (IIIA) in comparison with CsA group (IIA) (p3<0.001).

In contrast, there was no statistically significant difference between control group (IA) and group (CsA $\&$ Simvastatin) (IIIA) (p2=0.071).

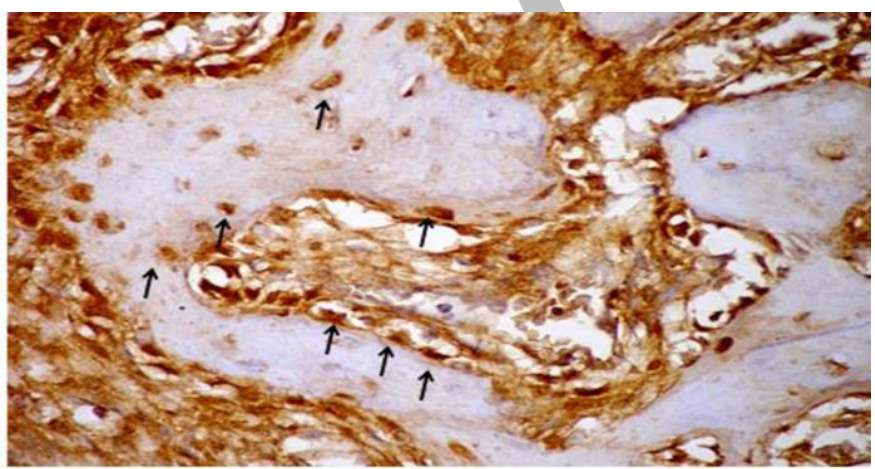

Fig. 6: Paraffin section micrograph of rat alveolar bone administrated (CsA\& Simvastatin 30 days) in the apical portion. (ABC $-\mathrm{DAB}$ Bar $=50 \mu \mathrm{m} \times 400)$
Table 1: Comparison between the control and the two studied groups according to bone volume.

\begin{tabular}{|c|c|c|c|c|c|c|}
\hline & \multicolumn{2}{|c|}{ Group I } & \multicolumn{2}{c|}{ Group II } & \multicolumn{3}{c|}{ Group III } \\
\hline & $\begin{array}{c}\text { A } \\
(n=5)\end{array}$ & $\begin{array}{c}\text { B } \\
(n=5)\end{array}$ & $\begin{array}{c}\text { A } \\
(n=5)\end{array}$ & $\begin{array}{c}\text { B } \\
(n=5)\end{array}$ & $\begin{array}{c}\text { A } \\
(n=5)\end{array}$ & $\begin{array}{c}\text { B } \\
(n=5)\end{array}$ \\
\hline Bone volume & & & & & & \\
\hline Min.-Max. & $0.17-0.23$ & $0.19-0.28$ & $0.09-0.15$ & $0.06-0.11$ & $0.20-0.33$ & $0.22-0.34$ \\
\hline Mean \pm SD. & $0.21 \pm 0.02$ & $0.23 \pm 0.04$ & $0.12 \pm 0.02$ & $0.09 \pm 0.02$ & $0.26 \pm 0.05$ & $0.29 \pm 0.06$ \\
\hline Median & 0.22 & 0.21 & 0.13 & 0.11 & 0.26 & 0.32 \\
\hline$P_{1}$ & & & $0.002^{*}$ & $<0.001^{\circ}$ & 0.071 & $0.040^{\circ}$ \\
\hline$P_{2}$ & & & & & $<0.001$ & $<0.001^{\circ}$ \\
\hline$P_{3}$ & 0.504 & 0.082 & & 0.424 \\
\hline
\end{tabular}

Table (1): Comparison between the different studied groups according to Bone volume $p_{1}: p$ value for Post Hoc Test (LSD) for companing between Group I and each other groups $p_{2}: p$ value for Post Hoc Test (LSD) for companing between Group II and Group III

$p_{3}: p$ value for Student t-test for comparing between $A$ and $B$ in each group

*: Statistically significant at $p \leq 0.05$

\section{Group B (after 30 days):}

Table (1) illustrated the comparison between control (IB), CsA (IIB), and (CsA \&Simvastatin) (IIIB) regarding bone volume in the apical region, where the values were $(0.23 \pm$ $0.04,0.09 \pm 0.02,0.29 \pm 0.06)$ respectively.

It was noticed that (CsA \&Simvastatin) (IIIB) exhibited the highest bone volume followed by the control group (IB), where the lowest bone volume related to CsA (IIB).

There was statistically significant decrease between CsA (IIB) and control (IB) (p1 <0.001), also a high significant increased between CsA (IIB), and (CsA \&Simvastatin) (IIIB) (p1<0.001).

On the other hand, after Simvastatin treatment there was a statistically significant difference increased in (CsA \&Simvastatin) (IIIB) compared to control (IB) group (p2= 0.040).

\section{b. Immunohistochemical Optical Density (IOD) of BMP 2: Group A (15 days)}

The mean value of IOD for the BMP 2 were $117.62 \pm 5.83$, $98.07 \pm 9.92$, and $121.51 \pm 1.68$ for group IA (control), IIA (CsA), and IIIA (CsA\& Simvastatin), respectively.

From table (2), there was a statistically significant decrease in IOD between group IA (control) and IIA (CsA), where $p$ value is $p 1<0.001$. Also, there was a statistically significant increase in IOD of BMP-2 for group IIIA (CsA\& simvastatin) than group IA (control), where $p$ value is p3 $<0.001$.

There was no statistically significant difference in IOD between group IA (control) and group IIIA (CsA\& simvastatin).

\section{Group B (30 days)}

The mean value of IOD for the BMP 2 in group B were $116.37 \pm 6.53,95.37 \pm 8.73$, and $95.37 \pm 8.73$ for group IB (control), IIB (CsA), and IIIB (CsA\& Simvastatin), respectively.

There was a statistically significant decrease in IOD 
between group IB (control) and IIB (CsA), where p value is $\mathrm{p} 1<0.001$. Also, there was a statistically significant increase in IOD of BMP-2 for group IIIB (CsA\& simvastatin) than group IB (control), where $\mathrm{p}$ value is $\mathrm{p} 3<0.001$.

There was no statistically significant difference in IOD between group IB (control) and group IIIB (CsA\& simvastatin).

Table 2: Comparison between the control and the two studied groups according to IOD of BMP 2 in the alveolar bone.

\begin{tabular}{|c|c|c|c|c|c|c|}
\hline & \multicolumn{2}{|c|}{ Groupl } & \multicolumn{2}{|c|}{ Group II } & \multicolumn{2}{|c|}{ Group III } \\
\hline & $\underset{(n=5)}{A}$ & $\begin{array}{c}B \\
(n=5)\end{array}$ & $\underset{(n=5)}{A}$ & $\begin{array}{c}B \\
(n=5)\end{array}$ & $\underset{(n=5)}{A}$ & $\begin{array}{c}B \\
(n=5)\end{array}$ \\
\hline \multicolumn{7}{|l|}{ Subtract } \\
\hline Min.-Max. & $110.3-125.59$ & $108.7-125.15$ & $82.75-107.7$ & $83.43-103.72$ & $118.99-124.0$ & $113.25-125.5$ \\
\hline Mean $\pm S D$. & $117.62 \pm 5.83$ & $116.37 \pm 6.53$ & $98.07 \pm 9.92$ & $95.37 \pm 8.73$ & $121.51 \pm 1.68$ & $119.0 \pm 5.15$ \\
\hline Median & 118.04 & 115.01 & 100.62 & 96.14 & 121.9 & 118.46 \\
\hline$P_{1}$ & & & $<0.001^{\circ}$ & $<0.001^{\circ}$ & 0.332 & 0.533 \\
\hline$P_{2}$ & & & & & $<0.001^{\circ}$ & $<0.001^{\circ}$ \\
\hline $\mathrm{p}_{3}$ & \multicolumn{2}{|c|}{0.724} & \multicolumn{2}{|c|}{0.627} & \multicolumn{2}{|c|}{0.348} \\
\hline
\end{tabular}

p1: $\mathrm{p}$ value for Post Hoc Test (LSD) comparing GroupI and each of the other groups.

p2: p value for Post Hoc Test (LSD) comparing GroupII and GroupIII

p3: $p$ value for Student t-test comparing between A and B in each group

*: Statistically significant

\section{DISCUSSION}

It is estimated that 200 million women have osteoporosis worldwide, for these reason it is considered as a major public health problem (24).

Both osteoporosis and periodontal diseases are bone resorptive diseases; it has been hypothesized that osteoporosis could be a risk factor for the progression of periodontal disease (9).

Immunosuppressive drugs that are used mainly after organ transplantation to prevent rejection as cyclosporine A, are associated with post transplantation-related osteoporosis (10).

Cyclosporin A (CsA) immunosuppressive properties result primarily from inhibition of $\mathrm{T}$ lymphocyte activation. CsA suppresses the immune response by inhibiting evolutionary conserved signal transduction pathways. CsA binds to their intracellular receptors, immunophilins, creating composite surfaces that block the activity of specific targets. For CsA/cyclophilin the target is calcineurin. Inhibition of the action of calcineurin results in a complete block in the translocation of the nuclear factor of activated $\mathrm{T}$ cells (NF-AT), resulting in a failure to activate the genes regulated by the NF-AT transcription factor. These genes include those required for B-cell help such as interleukin (IL-4) and CD40 ligand as well as those necessary for T-cell proliferation such as IL-2 (25).

In the present study, we used an established and well characterized animal model to evaluate, firstly, the role of simvastatin in the prevention of cyclosporine A-induced alveolar bone loss on the absence of inflammation and, secondly, estimate the expression of BMP-2 after simvastatin therapy indicating bone formation.

In this study, the duration of induction of alveolar bone loss by administration of immunosuppressive doses of cyclosporine A for 30 days caused alveolar bone loss, in agreement with previous reports $(26,27)$. They observed an increased bone resorption, represented by a significant increase in osteoclast-like cell number, accompanied by a reduction in the bone volume.

Cyclosporine A (Sandimmun_) was given subcutaneously daily (10 $\mathrm{mg} / \mathrm{kg}$ body weight, once a day).According to Wassef et al (20), this dosage provides plasma peak and therapeutic concentrations of 1000 and 750 $\mathrm{ng} / \mathrm{mL}$, respectively. This dosage of cyclosporine $\mathrm{A}$ and period of treatment were previously shown to cause alveolar bone loss in rats (28).

Simvastatin (Zocor) was given oral daily doses (once a day) at $20.0 \mathrm{mg} / \mathrm{kg}$. This method has been used successfully in previous studies in this animal model (21). The dose was within the range found to be safe and effective for increasing bone density in rats, whereas literature posology reports include both weekly and daily administrations (29).

In addition, we showed that administration of simvastatin counteracted the deleterious effects of cyclosporine A on bone turnover in the absence of inflammation. These results are compatible with those reported by Ohno et al (29), who showed that treatment with cerivastatin (a synthetic statin) improves cyclosporine Ainduced high-turnover osteopenia in transplanted bone, mainly through the inhibition of bone resorption. Many studies also stated the same results $(26,27,30)$.

Immunohistochemical analysis was done to study the expression of BMP 2 in all groups. The results of this study showed a significant increase in the BMP 2 after simvastatin administration, approximately near that in the control), while it showed a significant decrease in CsA treated group.

BMPs are synthesized as large precursors, which are processed and proteolytically cleaved to yield biologically active substance. BMPs have been demonstrated in osteoblasts, and osteoprogenitor cells, and surprisingly in osteoclasts (31).

McCullough et al conducted a study to examine the immunohistochemical distribution and intensity of staining of BMPs in various cell types found in the ectopic bone to define the role of BMPs in bone formation. The results was moderate to intense cytoplasmic immunostaining for BMP-2 in osteoprogenitor cells, most osteoblasts showed at least moderate staining for BMP-2 and There was moderate to intense staining for BMP-2 in most osteocytes found within the trabecular bone matrix (32).

In the present study, we found that the level of expression of BMP 2 in bone matrix, osteoblasts, and osteocytes in CsA group is significantly lower than that in the control. Luppen et al supported our result, They propose that glucocorticoid (GC), another immunosuppressive drug inhibited Bmp2, which was secondary to the inhibition of 
the osteoblast phenotype (33).

In simvastatin group, the level of expression of BMP-2 was significantly higher than in the CsA group, but to a near extent similar to the control. In agreement with Garrett et al (34).

BMP-2 has been shown to enhance collagen synthesis. In addition, BMP-2 induces a significant increase in cellular alkaline phosphatase activity at doses ranging between 20 and $200 \mathrm{mg} / \mathrm{ml}$ (35).

In agreement with Ohnaka et al, who stated that Pitavastatin, a newly developed statin, increased the expression level of mRNA for BMP-2 (36).

In bone volume histomorphometric analysis, we can thus state that CsA at a dose of $10 \mathrm{mg} /(\mathrm{kg}$ body weight day) leads to a significant decrease in bone volume per total volume (BV/TV). These results were supported by Nasser et al (37).

Animal studies have demonstrated acute increases in bone remodeling consisting of both osteoclast activation and, in some circumstances, increases in osteoblastic bone formation in CsA treated animals. There is limited but persuasive evidence that $\mathrm{T}$ cells may mediate the action of CsA (25).

While in simvastatin treated group the bone volume per total volume (BV/TV) were significantly increase. Junqueira et al supported our results and stated that there is a statistical significant differences were observed between the ovariectomized animals of the control and simvastatin treated groups, in histomophpmetric analysis of bone volume proving that treatment with simvastatin improved the bone regeneration of the ovariectomized rats (21).

Finally, it can be recommended to use statins in the treatment of osteoporotic patients, especially who receive immunosuppressive drugs.

\section{CONCLUSIONS}

Simvastatin counteracts the adverse effect of CsA induced alveolar bone loss by induction of BMP-2 in osteoblasts, and osteocytes that induces new bone formation. Also Simvastatin has a pleotropic effect as an anti-inflammatory, antioxidant, and anticancerous agent.

\section{CONFLICT OF INTEREST}

The authors declare that they have no conflict of interest.

\section{REFERENCES}

1. Kular J, Tickner J, etal. An overview of the regulation of bone remodelling at the cellular level. Clinical Biochemistry 2012; 863-873.

2. Sommerfeldt D., Rubin C. Biology of bone and how it orchestrates the form and function of the skeleton. Eur Spine J 2001; $10: 86-95$

3. Parfitt AM: Targeted and nontargeted bone remodeling: Relationship to basic multicellular unit origination and progression. Bone 2002; 30: 5-7.

4. Burr DB: Targeted and nontargeted remodeling. Bone 2002; 30: 2-4.

5. Ducy, P.; Karsenty, G. The family of bone morphogenetic proteins. Kidney Int. 2000; 2207-2214.

6. Urist, M.R. Bone: Formation by autoinduction. Science $1965 ; 150,893-899$.

7. Genant HK, Cooper C, Poor G, et al. Interim report and recommendations of the World Health Organization Task- Force for Osteoporosis. Osteoporos Int 1999; 10: 259.

8. National Osteoporosis Foundation. 2008. Avaliable from: www.nof.org/osteoporosis/diseasefacts.html.

9. Guiglia R., Di-Fede O., Lo-Russo L. Osteoporosis, jawbones and periodontal disease. Med Oral Patol Oral Cir Bucal. 2013; 18(1): 93-99.

10. Cohen A, Shane E. Osteoporosis after solid organ and bone marrow transplantation. Osteoporos Int 2003; 14:617-630.

11. David J., David P., Spiros Liras, and Price D. The Future of Peptide-based Drugs. Chem Biol Drug Des 2013; 81: 136-147.

12. Ming-Jen M., Fu E., Kuo P. Gelatinases and Extracellular Matrix Metalloproteinase Inducer Are Associated With Cyclosporin-A-Induced Attenuation of Periodontal Degradation in Rats. Journal of Periodontology January 2015; 82-90.

13. Dempster DW. Bone histomorphometry in glucocorticoidinduced osteoporosis. J Bone Miner Res 1989; 4: 137.

14. Alberts AW, Chen J, Kuron G, Hunt V, Huff J, Hoffman C, Rothrock J, Lopez M. Mevinolin: A highly potent competitive inhibitor of hydroxymethylglutarylcoenzyme A reductase and a cholesterol-lowering agent. Proceedings of the National Academy of Sciences of the USA. 1980; 77(7): 3957-3961.

15. Brunton LL, Lazo JS, Parker KL. Goodman and Gilman's The Pharmacological Basis of Therapeutics (11th edition). McGraw-Hill. 2007.

16. Kinra $P$, Khan S Simvastatin: Its potential new role in periodontal regeneration: Biology and Medicine. 2011; 215-221.

17. Mundy G, Garrett R, Harris S et al. Stimulation of bone formation in vitro and in rodents by statins. Science 1999; 286:1946-1949.

18. Edwards CJ, Spector TD. Statins as modulators of bone formation. Arthritis Res 2002; 4: 151-3.

19. Thurnher M., Nussbaumer O, Gruenbacher G. Novel Aspects of Mevalonate Pathway Inhibitors as Antitumor Agents. Clin Cancer Res July 1, 2012 vol. 18 no. 13 3524-3531.

20. Wassef R, Cohen Z, Langer B: Pharmakocinetic profiles of cyclosporine in rats. Influence of route of administration and dosage. Transplantation. 1985; 40:489-493.

21. Junqueira JC, Mancini MN, Carvalho YR, Anbinder AL, Balducci I, Rocha RF:Effects of simvastatin on bone regeneration in the mandibles of ovariectomized rats and on blood cholesterol levels. J Oral Sci 2002; 4:117-124.

22. Buchwalow IB, Bocker W.: immunohistochemistry: basic\& methods; page 33-58; Springer Verlag Berlin 
Heidenberg; 2010.

23. Svedbom A. Hernlund E. Ivergård M. Epidemiology and Economic Burden of Osteoporosis in Austria; A report prepared in collaboration with the International Osteoporosis Foundation (IOF) and the European Federation of Pharmaceutical Industry Associations (EFPIA). Arch Osteoporos. 2013; 8:137.

24. International Osteoporosis Foundation. The facts about osteoporosis and its impact. International Osteoporosis Foundation Web site. Available at: http://www.osteofound.org/press_centre/fact_ sheet.html. [Acccessed 26 July 2005.

25. Adina E., Mone Zaidi, Solomon E. Molecular Effects of Calcineurin Inhibitors. Bone Disease of Organ Transplantation, Chapter 5. 2005, Pages 79-89.

26. Spolidorio LC, Spolidorio DM, Holzhausen M. Effects of long-term cyclosporine on the periodontium of rats. $\mathrm{J}$ Periodont Res 2004; 39:257-262.

27. Spolidorio LC, Nassar PO, Nassar CA, Spolidorio DM, Muscara' MN. Conversion of immunosuppressive monotherapy from cyclosporin A to tacrolimus reverses bone loss in rats. Calcif Tissue Int 2007; 81:114-123

28. Stewart PJ, Stern PH. Cyclosporines: correlation of immunosuppressive activity and inhibition of bone resorption. Calcif Tissue Int 1989; 45:222-226.

29. Ohno T, Shigetomi M, Ihara $\mathrm{K}$ et al. Skeletal reconstruction by vascularized allogenic bone transplantation: effects of statin in rats. Transplantation 2003; 76:869-871.

30. Paula GOES1, Ana Patrícia Souza LIMA1, Iracema Matos. Effect of Atorvastatin in Radiographic Density on Alveolar Bone Loss in Wistar Rats. Braz Dent J 2010; 21(3): 193-198.

31. Anderson HC, Hodges PT, Aguilera XM. Bone morphogenetic protein (BMP) localization in developing human and rat growth plate, metaphysis, epiphysis, and articular cartilage. J Histochem Cytochem 2000; 48:1493-1502.

32. McCullough KA, Waits CA, Garimella R. Immunohistochemical Localization of Bone Morphogenetic Proteins (BMPs) 2, 4, 6, and 7 during Induced Heterotopic Bone Formation. J Orthop Res 2007; 25:465-472.

33. Luppen CA, Chandler RL, Frenkel B. BMP-2 vs. BMP4 expression and activity in glucocorticoid-arrested MC3T3-E1 osteoblasts: Smad signaling, not alkaline phosphatase activity, predicts rescue of mineralization. Growth Factors. 2008; 26(4): 226-237.

34. Garrett IR, Mundy GR. Commentary the role of statins as potential targets for bone formation. Arthritis Res 2002; 4:237-240.

35. Sugiyama M, Kodama T, Konishi K, Abe K, Asami S, Oikawa S. Compactin and simvastatin, but not pravastatin, induce bone morphogenetic protein-2 in human osteosarcoma cells. Biochemical and Biophysical Research Communications, 2000; 271(3): 688-692.
36. Ohnaka K. Shimoda S. Nawata H. Pitavastatin Enhanced BMP-2 and Osteocalcin Expression by Inhibition of Rho-Associated Kinase in Human Osteoblasts. Biochemical and Biophysical Research Communications 2001; 337-342.

37. NassarPO,NassarCA,Guimara esMR, AquinoSG, AndiaDC, MuscaraMN, Spolidorio DMP. Simvastatin therapy in cyclosporine A-induced alveolar bone loss in rats. J Periodont Res 2009; 44: 479-488.

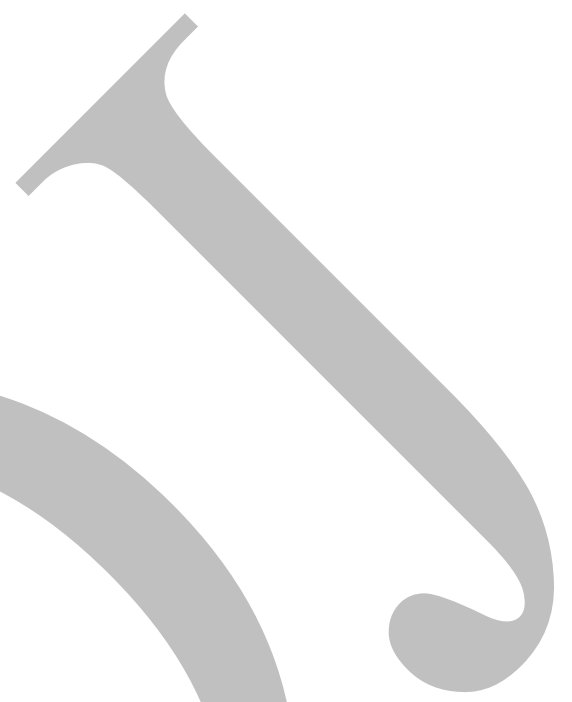

